\title{
Filière des produits à usage médicinal issus de Cordyla pinnata, Detarium microcarpum et Detarium senegalense au Sénégal
}

\author{
Received: 2019-11-15; revised: 2019-12-12; accepted: 2019-12-17
}

\author{
Sara Danièle Dieng ${ }^{1 *}$, Fatimata Niang-Diop ${ }^{1}$, Mamadou Diop ${ }^{1}$, Assane Goudiaby ${ }^{1}$, Bienvenu Sambou ${ }^{1}$, Anne \\ Mette Lykke ${ }^{2}$
}

\author{
1. Institut des Sciences de l'Environnement, Université Cheikh Anta Diop de Dakar, Dakar-Fann, Sénégal \\ 2. Department of Bioscience, Aarhus University, Silkeborg, Denmark \\ * Auteur correspondant; adresse Email: saradieng@gmail.com
}

\begin{abstract}
Résumé: Cette étude avait pour objectifs: 1) d'identifier et de caractériser les acteurs impliqués dans la filière des produits à usage médicinal tirés de Cordyla pinnata, Detarium microcarpum et Detarium senegalense au Sénégal; 2) d'analyser l'organisation de cette filière et l'importance des parties utilisées; 3) d'analyser la commercialisation de ces produits; 4) d'identifier les forces, faiblesses, opportunités et menaces de la filière. L'échantillonnage en boule de neige a été utilisé et a permis d'interroger, à l'aide de guides d'entretien 13 tradipraticiens locaux, 3 récolteurs et 17 herboristes. Les résultats ont révélé que les acteurs de cette filière, majoritairement des hommes, entretenaient des relations de coopération et qu'ils bénéficiaient de l'appui de la société civile en termes de formation et d'organisation. Les racines et les écorces de Cordyla pinnata et Detarium microcarpum étaient plus utilisées tandis que celles de Detarium senegalense étaient peu commercialisées. Les écorces, les racines et les feuilles étaient vendues entre 0,108 et 0,290 Euro/100g. Une partie des écorces était transformée en poudre avant d'être commercialisée chez les herboristes entre 1,046 Euro/100g (pour Cordyla pinnata) et 1,524 Euro/100g (Detarium microcarpum). Les prix étaient plus élevés à Dakar. Les parties tirées de Cordyla pinnata étaient essentiellement utilisés contre les parasitoses intestinales tandis que celles de Detarium microcarpum et dans une moindre mesure celles de Detarium senegalense servaient à soigner les dermatoses. Les faiblesses de cette filière concernaient essentiellement l'exploitation anarchique de la ressource et la raréfaction saisonnière de certains produits tandis que l'éloignement des sites d'approvisionnement menaçait son bon fonctionnement.
\end{abstract}

Mots clés: Cordyla pinnata, Detarium microcarpum, Detarium senegalense, filière, produits à usage médicinal, Sénégal.

\section{VALUE CHAIN OF PRODUCTS FOR MEDICINAL USE DERIVED FROM CORDYLA PINNATA, DETARIUM MICRO- CARPUM AND DETARIUM SENEGALENSE IN SENEGAL}

Summary: The objectives of this study were: 1) to identify and characterize actors involved in products for medicinal value chain of Cordyla pinnata, Detarium microcarpum and Detarium senegalense in Senegal; 2) to analyze the organization of this value chain and the importance of plant parts used; 3) to analyze the commercialization of these products; 4) to identify the strengths, weaknesses, opportunities and threats of the value chain. Snowball sampling was used and allowed to interview, using interviews guides, 13 local healers, 3 harvesters and 17 herbalists. Results showed that actors involved in this value chain collaborated together and benefit from the support of civil society in terms of training and organization. The roots and bark of Cordyla pinnata and Detarium microcarpum were the most widely used while those of Detarium senegalense were poorly commercialized. Bark, roots and leaves were commercialized between 0.108 and 0.290 Euro/100g. Part of the bark was transformed into powder before being marketed among herbalists between 1.046 Euro/100g (for Cordyla pinnata) and 1.524 Euro/100g (Detarium microcarpum). Selling prices were higher in Dakar. Plant parts derived from Cordyla pinnata were mainly used against intestinal parasites while those of Detarium microcarpum and, to a lesser extent, those of Detarium senegalense were used to treat dermatosis. This value chain was faced with weaknesses such as uncontrolled exploitation of the resource and the seasonal scarcity of certain products while long distance from supply sites threatened its good functioning.

Keywords: Cordyla pinnata, Detarium microcarpum, Detarium senegalense, products for medicinal use, Senegal, value chain. 


\section{INTRODUCTION}

Les écosystèmes naturels fournissent aux populations des ressources utilisées à des fins diverses: alimentaires, artisanales, médicales et spirituelles (LYKKE \& al. 2004, IPBES 2019). Quatre milliards de personnes dans le monde dépendent des médecines naturelles pour leur santé (IPBES 2019). En Afrique, les plantes occupent une place de choix dans la santé humaine (Assogbadjo \& al. 2010, GueYe \& al. 2012, WitTig \& al. 2013). Dans des pays comme le Bénin et le Burkina Faso, plus de 30\% des plantes vasculaires sont utilisées à titre aromatique ou médicinal (SIEGLSTETTER $\&$ al. 2011, WitTig \& al. 2013). En plus de leur utilisation dans la médecine traditionnelle, de nombreux extraits de plantes sont utilisés dans l'industrie pharmaceutique pour la fabrication de médicaments modernes et font l'objet d'un commerce international (CUNNINGHAM \& MBENKUM 1993, Coppen 1995). Malgré l'importance économique des plantes dans la santé humaine, peu d'études s'intéressent aux filières des produits à usage médicinal d'origine végétale (VAdouhê \& al. 2008, Booker \& al. 2012). Parmi celles qui concernent l'Afrique, la plupart des études se concentrent sur le Formule 2l'analyse de leurs caractéristiques chimiques (DE WET \& VAN WYK 2007, BaKWAYE $\&$ al. 2013, DiATtA \& al. 2013, Dibong \& al. 2015, FALL \& al. 2017). Toutefois, le profil des personnes qui utilisent ces médicaments, les sources d'approvisionnement ainsi que l'organisation de la filière créée à partir de leur commercialisation restent pour la plupart peu connus (ALBUQUERQUE \& al. 2007). Ces éléments nous amènent à étudier la filière des produits à usage médicinal tirés de trois espèces ligneuses de grande importance socio-économique dans la zone soudanienne sénégalaise. Il s'agit de Cordyla pinnata, Detarium microcarpum et Detarium senegalense. Cette étude, qui s'intéresse aux maillons de la récolte et de la commercialisation, se fixe comme objectifs: 1) d'identifier et de caractériser les acteurs impliqués dans la filière de ces produits à usage médicinal; 2) d'analyser l'organisation de cette filière et l'importance des parties utilisées, 3) d'analyser la commercialisation de ces produits; et 4) d'identifier les forces, les faiblesses, les opportunités et les menaces de la filière.

\section{MATÉRIEL ET MÉTHOdE}

\subsection{Zone d'étude}

La présente étude a été réalisée dans trois sites: 1) la Forêt Classée de Patako et sa périphérie, 2) des marchés des centres urbains de Kaolack et 3) de Dakar (Fig. 1).

La Forêt Classée de Patako est localisée entre $13^{\circ} 40^{\prime}$ et $16^{\circ} 15^{\prime}$ Ouest, dans la région de Fatick. Le climat est marqué par l'alternance d'une saison sèche de novembre à juin et d'une saison pluvieuse, de juillet à octobre. La moyenne pluviométrique dans la période de 1983 à 2013 était de 753 mm (DiENG 2017). Les types de végétation présentes dans cette forêt sont la savane arborée à arbustive, la savane boisée à proximité des vallées, la forêt galerie dans les vallées et la mangrove dans les zones humides saumâtres (SAMBOU 2004). La population est majoritairement constituée de wolofs (plus de 60\%) et de mandingues (environ 18\%). L'agriculture est la principale activité socio-économique avec comme principales cultures le mil, l'arachide, le maïs et le sorgho.

La ville de Kaolack, située au cœur du Bassin Arachidier, au centre-ouest du Sénégal et à environ $95 \mathrm{~km}$ de la Forêt Classée de Patako, joue un rôle important dans le commerce intérieur et extérieur du Sénégal. Sa position centrale en fait un passage obligé vers les régions nord et sud du pays et vers les autres pays limitrophes (Gambie, Guinée Bissau, Guinée Conakry et Mali). Trois marchés ont été visités pour la collecte des données. Le marché central et le marché Guedj sont situés au quartier Léona entre $14^{\circ} 13^{\prime}$ Nord et $16^{\circ} 07^{\prime}$ Ouest. Le marché Médina, pour sa part, est situé entre $14^{\circ} 09$ Nord et $16^{\circ} 03^{\prime}$ Ouest, dans le quartier de Médina Baye.

La région de Dakar est située dans la presqu'île du Cap Vert et couvre une superficie de 550 kilomètres carrés. Située à environ $260 \mathrm{~km}$ de la Forêt Classée de Patako, cette région comptait 3137196 habitants en 2013 (ANSD 2015). L'activité commerciale y est dominée par le secteur informel qui est essentiellement animé par des personnes issues des zones rurales. Un marché a été visité à Dakar. Il s'agit du marché Tilène, situé entre $14^{\circ} 68^{\prime}$ Nord et $17^{\circ} 44^{\prime}$ Ouest dans la commune de la Médina, département de Dakar.

\subsection{Matériel d'étude}

Cordyla pinnata (A.Rich.) Milne-Redh. est un arbre de la famille des Fabacées qui pousse dans les savanes soudaniennes et les forêts sèches (Kerharo \& AdAm 1974, ArbonNIER 2019). Son fruit est une baie charnue, de forme plus ou moins sphérique et qui devient jaune à maturité. Dans la Forêt Classée de Patako, sa densité moyenne est estimée à 9 individus à l'hectare (SAMBOU 2004) contre 1 individu à l'hectare en zone de terroir périphérique (CHRISTENSEN 2010). Cordyla pinnata est la principale espèce agroforestière de la zone (Dıop \& al. 2012). Son caractère multi-usages dans la zone d'étude (DiEng \& al. 2016) justifie que l'on s'intéresse davantage à ses usages dans la médecine traditionnelle qui peuvent être des facteurs de dégradation du peuplement suivant la partie de la plante utilisée et l'intensité dans les prélèvements.

Detarium microcarpum Guill. \& Perr. est aussi un arbre de la famille des Fabacées (Fig. 2). Ses fruits charnus sont ovoïdes et contiennent une pulpe farineuse et verdâtre. Son aire de répartition s'étend de l'axe Sénégal-Cameroun jusqu'au Soudan. Detarium microcarpum fait l'objet de différentes utilisations. Son fruit, ses feuilles et ses fleurs entrent dans l'alimentation, tandis que ses feuilles, écorces, racines, amandes, entrent dans la médecine traditionnelle (Arbonnier, 2019, Kouyaté 2005). Dans la Forêt Classée de Patako, Detarium microcarpum a une population stable, marquée par une abondante régénération naturelle (SAMBOU, 2004). En 2004, sa densité moyenne a été évaluée à 3 individus à l'hectare (SAmbou, 2004). Elle est peu présente en zone de terroir. L'espèce fait l'objet d'une exploitation plus ou moins anarchique dans la zone, guidée notamment par ses propriétés thérapeutiques; ce qui justifie son choix dans cette étude. 


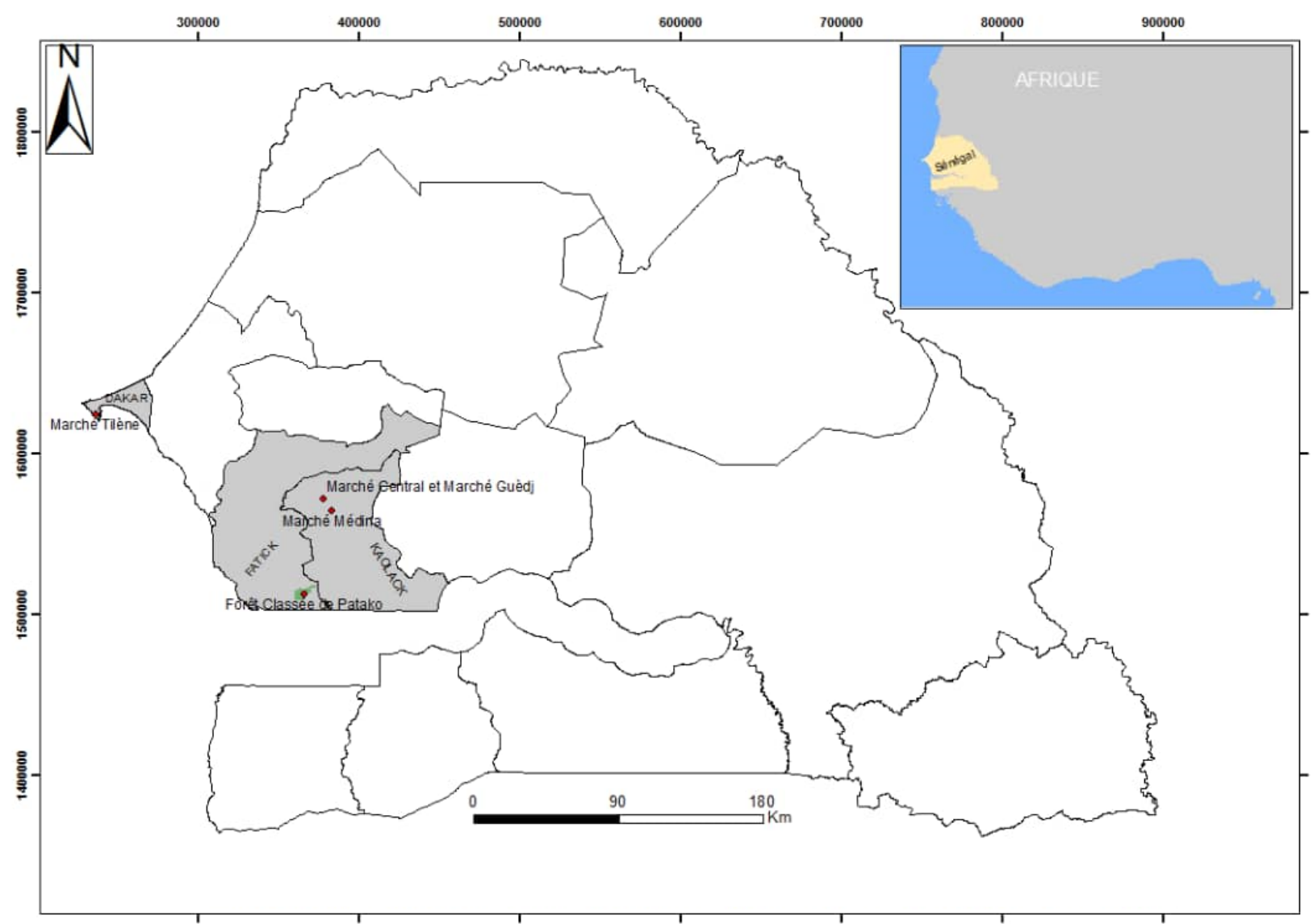

Fig. 1: Localisation des sites d'étude/ Location of study sites/ Standort der Untersuchungsorte .

Detarium senegalense J.F. Gmel., un arbre fruitier de la même famille que Detarium microcarpum, a une aire de répartition qui s'étend du Sénégal au Soudan (RousSEL 1995, ARBonNier 2019). Dans la zone couverte par la présente étude, 1'espèce est localisée le long des vallées à l'intérieur de la Forêt Classée de Patako et dans sa périphérie. Cependant, il n'existe pas encore d'informations quantitatives sur les caractéristiques de son peuplement dans la zone d'étude. Detarium senegalense est surtout exploité pour ses fruits riches en vitamine $\mathrm{C}$. Certains individus de Detarium senegalense produisent des fruits toxiques, sans que l'on puisse les différencier scientifiquement (KERHARO 1974, Roussel 1995, Diop 2013). Cependant, les populations locales parviennent à les identifier à l'aide d'indicateurs tels que l'emplacement de l'arbre, l'abondance des fruits non consommés sous son houppier et la saveur du fruit. L'exploitation des fruits est généralement marquée par de mauvaises pratiques dans la zone d'étude (cueillette de fruits non murs et élagage des arbres). En plus des fruits, certaines parties de l'arbre sont utilisées dans la médecine traditionnelle par les populations locales. D'où l'importance d'étudier l'intérêt médicinal de Detarium senegalense en vue de déterminer si, en dehors de la pression due à la cueillette des fruits, l'exploitation des autres parties de l'arbre constitue une menace pour la pérennité du peuplement.

\subsection{Méthodes et outils de collecte des données}

Cette étude s'intéresse aux personnes qui s'activent dans l'usage médicinal des plantes en milieu rural et dans cer-
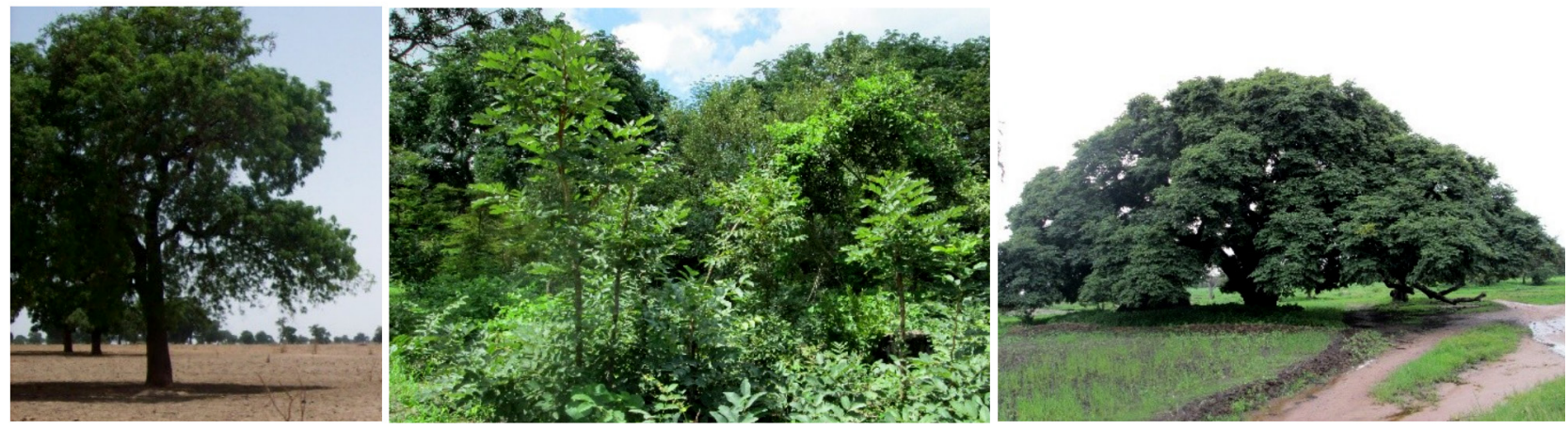

Fig. 2: Individus de Cordyla pinnata, Detarium microcarpum et Detarium senegalense/ Individuals of Cordyla pinnata, Detarium microcarpum and Detarium senegalense/ Individuen von Cordyla pinnata, Detarium microcarpum und Detarium senegalense. 
tains sites de commercialisation en zone urbaine. Il s'agit des guérisseurs traditionnels appelés 'tradipraticiens', des récolteurs et des herboristes.

La méthode d'identification des personnes à enquêter repose sur l'échantillonnage en boule de neige (Russel 2006, MARTín-LóPEZ \& al. 2011). Elle consiste à impliquer le (ou les) premiers contacts rencontrés dans l'identification des prochaines personnes à enquêter. Cette méthode est généralement utilisée pour les études de filières et celles portant sur des populations mobiles, dispersées ou difficiles à situer (Russel 2006).

Le choix des villages a été effectué à l'issue d'entretiens avec des personnes ressources. Ces dernières nous ont permis de localiser les villages où nous étions susceptibles de trouver le plus de tradipraticiens et plus spécifiquement le plus de phytothérapeutes. Dans chaque village, le chef de village et une personne ressource s'activant dans la médecine traditionnelle ont été mis à contribution pour identifier les acteurs qui sont impliqués dans cette activité. En fonction de la disponibilité de chacun et de sa disposition à participer à l'enquête, un entretien semi-structuré a été réalisé. Un total de 13 tradipraticiens et 2 récolteurs ont ainsi été interviewés à l'aide d'un guide d'entretien dans neuf localités situées dans un rayon de 5 kilomètres autour de la Forêt Classée de Patako. Des visites dans cette forêt et ses alentours ont permis d'observer des traces d'exploitation sur certains arbres.

A Kaolack, la même procédure a été utilisée, cette fois-ci avec l'aide du président de l'association des herboristes de la ville pour l'identification des premières personnes à interroger. Sur cette base, 12 herboristes et 1 récolteur ont pu être interrogés en différents endroits. A Dakar, 5 herboristes ont participé à l'enquête (Tableau 1).

Tableau 1: Répartition spatiale des acteurs interrogés/ Spatial distribution of the interviewed actors.

\begin{tabular}{|l|l|l|l|l|}
\hline Acteurs & $\begin{array}{l}\text { Périphérie FC } \\
\text { de Patako }\end{array}$ & Kaolack & Dakar & Total \\
\hline Tradipraticiens & 13 & 0 & 0 & 13 \\
\hline Récolteurs & 2 & 1 & 0 & 3 \\
\hline Herboristes & 0 & 12 & 5 & 17 \\
\hline Total & 15 & 14 & 5 & 33 \\
\hline
\end{tabular}

Les questions évoquées avec les différents acteurs de la filière concernaient: la spécialité, les utilisations médicinales des parties des trois espèces, l'approvisionnement, la commercialisation, les prix d'achat et de vente ainsi que les contraintes.

Un échantillon des différentes parties commercialisées chez trois herboristes a été acheté et pesé à l'aide d'une balance électronique à précision de marque Sartorius 1205 afin d'estimer le poids des bottes et de la poudre vendues.

\subsection{Traitement des données}

La cartographie fonctionnelle des acteurs de la filière a été réalisée suivant le modèle de GTZ (2007). Les données collectées ont fait l'objet d'un dépouillement. Pour cha- que informateur, un score de 1 a été affecté à chaque usage ou partie et celui de 0 en cas d'absence d'utilisation. L'importance relative des plantes étudiées a été estimée à partir d'indicateurs tels que la fréquence d'utilisation (F: Formule 1) des parties de chacune des plantes et la valeur consensuelle sur les types de maladies (CTU, inspirée de MonTeIro 2006; Formule 2) utilisée pour évaluer le degré de consensus pour les parties exploitées et les maladies soignées (Heinrich \& al. 1998, Monteiro 2006, SchumanN \& al. 2012, KABORÉ \& al. 2014).

$$
\text { Formule } 1 \quad F=\left(\frac{S}{N}\right) 100
$$

avec S: nombre d'informateurs qui ont répondu positivement à l'usage d'un partie et N: nombre total d'informateurs.

$$
\text { Formule } 2 \quad C T U=\left(\frac{T U}{U t}\right) / S
$$

avec TU: nombre de citations pour une maladie donnée; Ut: Nombre total de maladies citées par les acteurs et $\mathrm{S}$ : nombre de catégories de maladies auxquelles appartiennent les maladies citées.

L'ensemble des maladies citées ont été classées dans 9 groupes inspirés de la classification internationale des maladies (ATIH, 2018). Ces maladies sont liées: 1) au système digestif; 2) à la peau et aux tissus cellulaires; 3) au système génito-urinaire; 4) au système ostéo-articulaire; 5) aux maladies parasitaires et infectieuses; 6) aux signes et symptômes anormaux; 7) à la prévention; 8) aux maladies endocriniennes, nutritionnelles et métaboliques et 9) aux autres maladies qui enregistrent chacune 1 citation.

\section{RÉSULTATS}

\subsection{Les acteurs de la filière}

Les principaux acteurs de la filière de commercialisation des produits à usage médicinale tirés de Cordyla pinnata, Detarium microcarpum et Detarium senegalense sont constitués par les tradipraticiens et les récolteurs au niveau local, et les herboristes actifs dans la commercialisation en zone urbaine (Fig. 3).

Les tradipraticiens interrogés sont tous des hommes, âgés en moyenne de 57 ans avec un minimum de 40 ans et un maximum de 75 ans. En plus de maîtriser les vertus médicinales des espèces végétales, ils disposent généralement de connaissances sur les autres ressources naturelles pouvant soigner (espèces animales, eau et terre). Il y a souvent une dose de mysticisme et de secret dans leur travail, ce qui explique qu'ils soient parfois réticents à parler de leur métier. Ces tradipraticiens ont habituellement une spécialité pour laquelle ils sont reconnus par la communauté; même s'ils peuvent soigner d'autres maux. Ils se chargent généralement de la récolte des produits qu'ils utilisent dans leur activité et exercent leur métier à domicile avec des déplacements ponctuels dans d'autres localités du Sénégal et de la Gambie sur la demande de clients.

La récolte des produits à usage médicinal implique aussi bien les hommes que les femmes. Les premiers récoltent les racines et les écorces tandis que les femmes sont plutôt spé- 

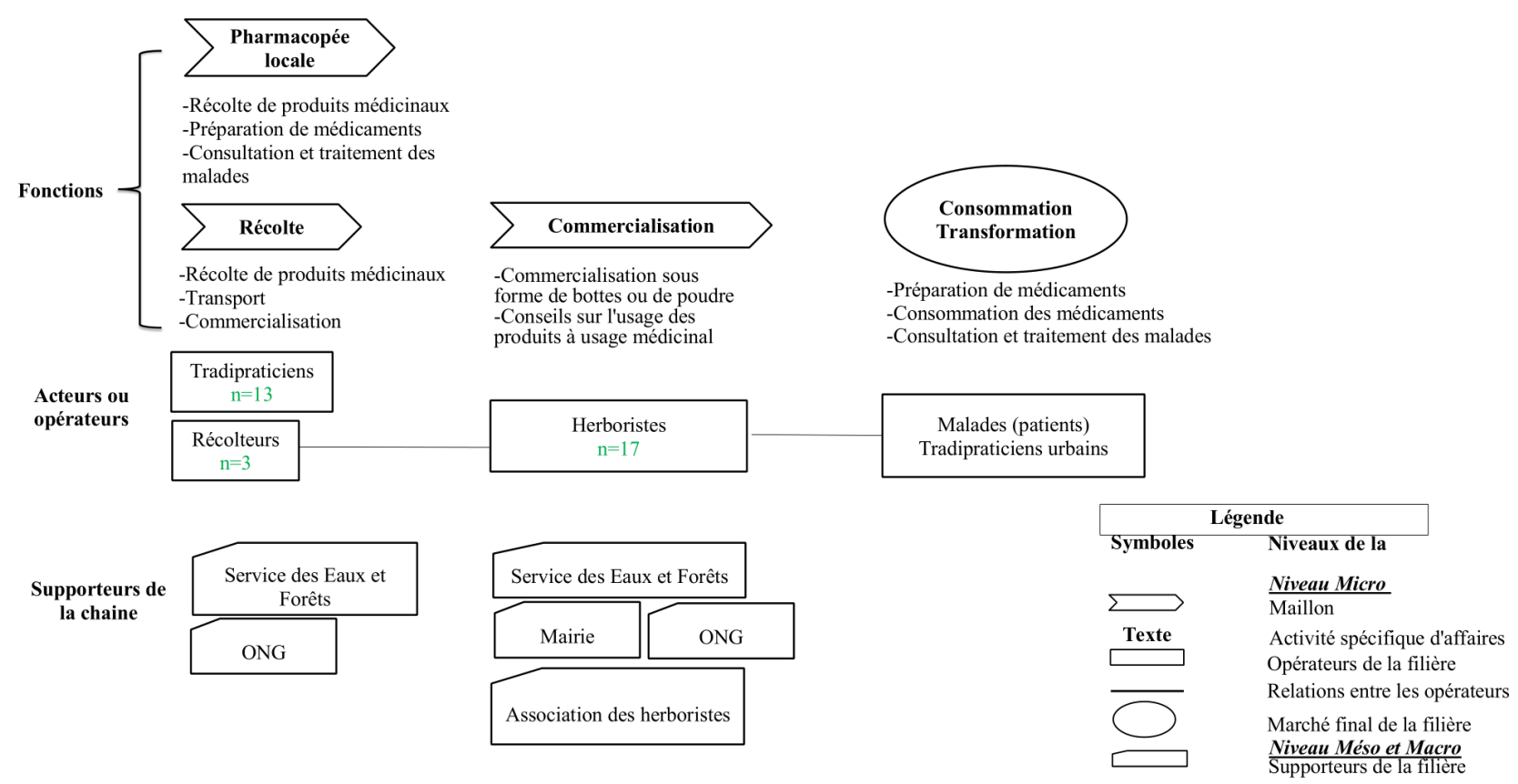

Fig. 3: Carte fonctionnelle des acteurs de la filière des produits médicinal tirés de Cordyla pinnata, Detarium microcarpum et Detarium senegalense/ Fonctional map of actors involved in the products for medicinal use value chain from Cordyla pinnata, Detarium microcarpum and Detarium senegalense/ Funktionsschema der Akteure im Arzneimittelsektor aus Cordyla pinnata, Detarium microcarpum und Detarium senegalense.

cialisées dans la récolte et la vente des feuilles. Les trois récolteurs interrogés sont des hommes âgés de 40 à 60 ans. Ils se chargent de récolter ces produits sur la demande de tradipraticiens et d'herboristes établis en ville ou pour les commercialiser eux-mêmes. Dans la Forêt Classée de Patako, l'activité est généralement clandestine car contraire au code forestier et les jours de récolte sont souvent influencés par les marchés hebdomadaires où sont échangés ces produits. Ces acteurs sont rémunérés soit en espèces, soit en nature. Dans ce dernier cas, des connaissances sur les vertus des plantes sont livrées aux récolteurs par les commanditaires. A côté des autochtones riverains de la Forêt Classée de Patako, d'autres acteurs habitant la périphérie des grandes villes telles que Kaolack en font une activité lucrative pendant la saison sèche. Ce sont essentiellement eux qui approvisionnent les herboristes en zone urbaine.

Les herboristes interrogés dans les marchés de Kaolack et Dakar sont essentiellement composés d'hommes et leur âge est compris entre 35 et 70 ans. La majorité des acteurs impliqués dans la commercialisation des produits à usage médicinal des trois espèces sont soit d'ethnie mandingue et dialonké et originaires du Mali, soit ils sont wolof. Les peuls et les sérères sont peu représentés. Seules deux femmes ont été identifiées et interrogées à Kaolack dans ce maillon. Si les hommes se consacrent généralement à temps plein dans la commercialisation des produits végétaux à usage médicinal, les femmes pour leur part, vendent plusieurs produits [tels que les fleurs de Hibiscus sabdarifa (bissap), les fruits de Adansonia digitata (baobab), la pâte d'arachide] à la fois; ce qui fait qu'elles ne remplissent pas toutes les conditions pour être considérées à part entière comme des herboristes. Cependant, avec l'accompagnement d'organisations de la société civile telles que Enda Santé, elles tendent à se spécialiser dans la commercialisation des produits végétaux à usage médicinal et font partie de l'association des herbo- ristes de Kaolack. Chaque herboriste a des fournisseurs fixes dans différentes localités du pays, chez qui il fait ses commandes. En cas de raréfaction de certains produits, il peut se rabattre sur ses collègues ou sur les récolteurs occasionnels qui sillonnent le marché pour écouler leurs produits.

La gouvernance de la filière des produits à usage médicinal, de façon générale, laisse apparaître deux niveaux: le niveau interpersonnel et le niveau institutionnel. Le premier niveau concerne les relations entre acteurs dans la récolte et la commercialisation des produits à usage médicinal, où des rapports de confiance et gagnant-gagnants sont établis. Au plan institutionnel, des organisations de la société civile appuient la filière à travers l'encadrement sur les techniques de récolte, d'entreposage et de conservation ainsi que dans le renforcement des capacités organisationnelles. Il s'y ajoute le rôle des services techniques, notamment celui des Eaux et Forêts qui se charge de veiller à la conformité des prélèvements avec les lois en vigueur, de contrôler et de recouvrir les taxes sur les produits prélevés. Les agents des Eaux et Forêts interviennent aussi bien dans les zones de prélèvement que dans les zones de débarquement des produits.

\subsection{Organisation de la filière et importance des parties utilisées}

L'analyse porte d'abord sur l'organisation de la filière des produits à usage médicinal tirés de Cordyla pinnata, Detarium microcarpum et Detarium senegalense en termes de lieu de collecte, de fréquences et de quantités, ensuite sur l'importance des parties utilisées et enfin sur leur commercialisation. 


\subsubsection{Organisation de la filière}

La récolte des produits à usage médicinal comporte deux périodes: une période intense pendant la saison sèche (novembre à mai) et une période marquée par des approvisionnements sporadiques en saison des pluies (juin à octobre). La période intense coïncide avec la fin des travaux champêtres et l'amenuisement progressif des ressources des paysans qui doivent se tourner vers d'autres activités lucratives pour subvenir aux besoins de la famille et assurer l'éducation des enfants. Pour certaines espèces comme Cordyla pinnata, l'approvisionnement se fait généralement en zone de terroir alors que pour Detarium microcarpum, il faut souvent aller dans les aires protégées pour s'en procurer. Cependant, certains récolteurs et tradipraticiens parviennent à s'en procurer en zone de terroir. Concernant cette dernière espèce, toutes les catégories d'acteurs interrogées conviennent qu'il est devenu de plus en plus difficile de trouver de grands sujets de Detarium microcarpum susceptibles d'être écorcés et que les sites d'approvisionnement sont de plus en plus éloignés. Les prélèvements de produits issus de Detarium senegalense, se font aussi bien en zone de terroir que dans les aires protégées (Fig. 4). Cette période intense coïncide également avec la fructification et la maturation des fruits de ces espèces.

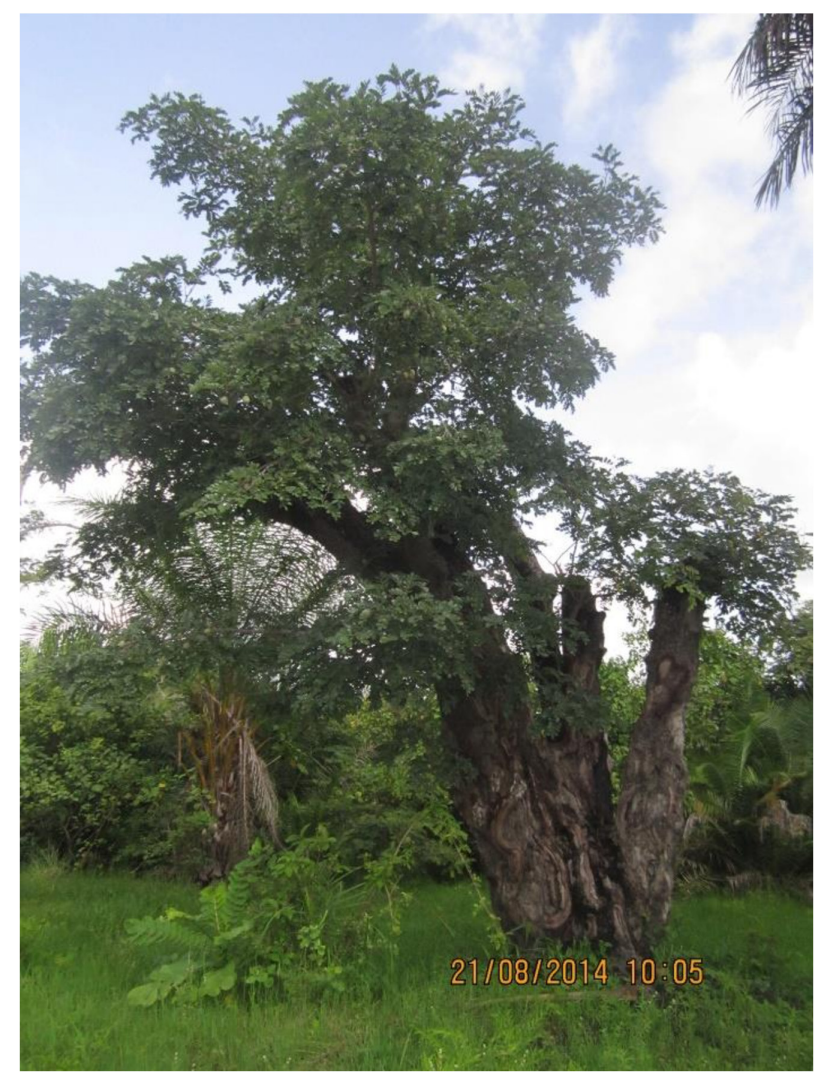

Fig. 4: Traces d'écorçage sur un individu de Detarium senegalense dans la zone d'étude/Traces from debarking on an individual of Detarium senegalense in the study area/ Rindenspuren an einer Individuum aus Detarium senegalense im Terroirbereich.

La période de moindre intensité dans la récolte correspond à la saison des pluies. A ce moment, la plupart des récolteurs sont occupés avec les travaux des champs tandis que les prélèvements des écorces et racines de Cordyla pinnata sont gênés par la présence des paysans dans leurs champs.
Il s'en suit des situations de pénurie chez les herboristes qui sont souvent obligés d'attendre plusieurs semaines avant de recevoir leurs commandes.

Néanmoins, la commercialisation est effectuée pendant toute l'année tandis que la fréquence d'approvisionnement dépend de l'offre et de la demande. Les herboristes s'approvisionnent en produits issus de de Cordyla pinnata une à deux fois par mois alors que l'approvisionnement est hebdomadaire dans la plupart des cas pour ceux de Detarium microcarpum; ce qui traduit un intérêt plus poussé des populations vers cette dernière espèce. L'approvisionnement en ce qui concerne de Detarium senegalense se fait mensuellement car la demande est faible par rapport aux deux autres espèces.

\subsubsection{Importance des parties de Cordyla pinnata, Detari- um microcarpum et Detarium senegalense}

Les produits issus de Cordyla pinnata, Detarium microcarpum et Detarium senegalense sont utilisés, à des degrés divers par toutes les catégories d'acteurs. Cependant, les parties tirées de Detarium microcarpum et Cordyla pinnata sont les plus utilisés aussi bien dans la pharmacopée locale que dans les sites de commercialisation. Les plus fortes fréquences d'utilisation sont notées pour les écorces de ces deux espèces pour lesquelles des valeurs supérieures à $80 \%$ et $90 \%$ sont observées respectivement chez les tradipraticiens et les herboristes (Fig. 5). Les racines de Detarium microcarpum sont également bien représentées dans l'activité des tradipraticiens et celle des herboristes avec des fréquences d'utilisation supérieures à $80 \%$. Les fréquences d'utilisation des feuilles sont plus faibles que celles des écorces et des racines avec des valeurs plus élevées chez les
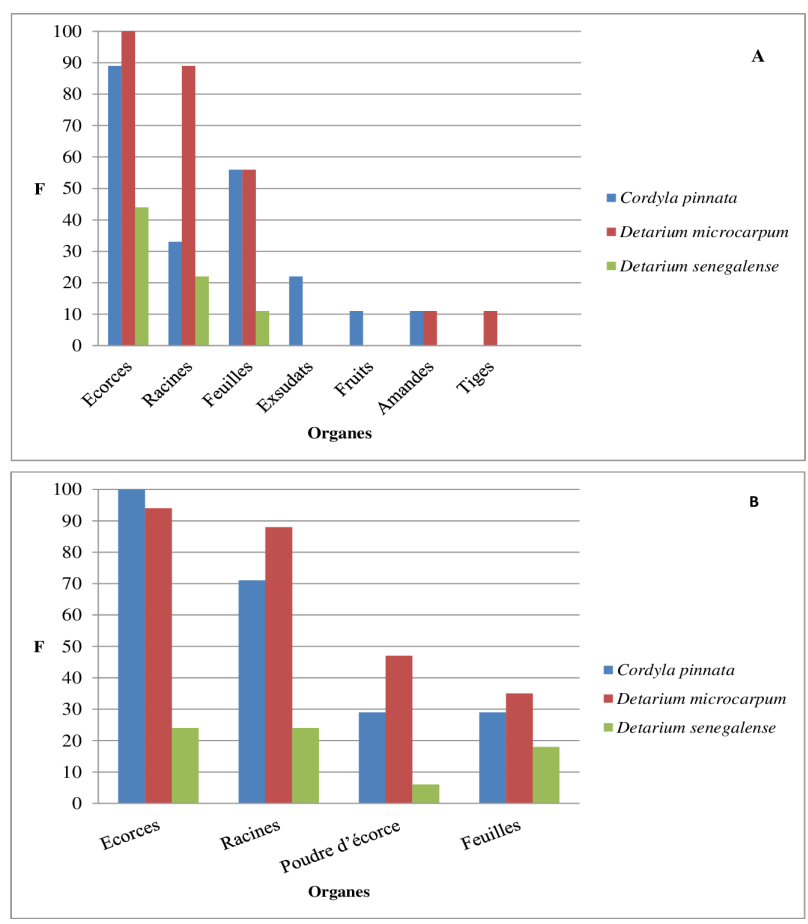

Fig. 5: Fréquences d'utilisation des parties des trois espèces chez les tradipraticiens (A) et chez les herboristes (B)/ Use frequency of parts of the three species by (A) traditional healers and (B) herbalists/ Häufigkeit der Nutzung von Organen der drei Arten durch traditionelle Heiler (A) und Kräuterkundige (B). 
tradipraticiens (supérieures à 50\%) que chez les herboristes (inférieures à $40 \%$ ). Contrairement à Cordyla pinnata et Detarium microcarpum, les produits tirés de Detarium senegalense sont peu utilisés par les tradipraticiens et les herboristes. Pour cette dernière espèce, nos investigations montrent que c'est la variété toxique qui est surtout utilisée dans la médecine traditionnelle.

En dehors des écorces, des racines et des feuilles, les tradipraticiens emploient d'autres parties issues de ces trois espèces pour exercer leur activité tandis que les herboristes commercialisent beaucoup la poudre obtenue à partir des écorces (Fig. 5).

Les applications médicinales de Cordyla pinnata et Detarium microcarpum sont plus diversifiées que celles de Detarium senegalense (Tableau 2). Cordyla pinnata et Detarium microcarpum sont plus reconnues pour leur action spéci- fique contre un type d'affection alors que pour Detarium senegalense tel n'est pas le cas. Les degrés de consensus les plus élevés sont observés chez les herboristes pour l'action anti-parasitaire de Cordyla pinnata (CTU=0,39), l'action antifongique de Detarium microcarpum qui est utilisé contre les dermatoses $(\mathrm{CTU}=0,64)$ et pour les problèmes de nerfs $(\mathrm{CTU}=0,16)$. La seule utilisation médicinale identifiée par les herboristes pour Detarium senegalense concerne son utilisation contre les dermatoses $(\mathrm{CTU}=0,11)$. Les tradipraticiens s'accordent davantage sur le rôle de Detarium microcarpum dans le traitement des dermatoses $(\mathrm{CTU}=0,12)$.

\subsubsection{Commercialisation des produits tirés de Cordyla pinnata, Detarium microcarpum et Detarium senegalense}

Les tradipraticiens et les herboristes reçoivent tous les types de clients quel que soit l'âge, l'ethnie, le sexe ou bien

Tableau 2: Principales utilisations médicinales de Cordyla pinnata, Detarium microcarpum et Detarium senegalense identifiées par les acteurs de la filière/Table 2: Main medicinal uses of Cordyla pinnata, Detarium microcarpum and Detarium senegalense identified by the actors of the value chain

\begin{tabular}{|c|c|c|c|c|c|c|}
\hline \multirow[t]{3}{*}{ Maladies/affections } & \multicolumn{6}{|c|}{ Valeur consensuelle sur les types de maladies (CTU) } \\
\hline & \multicolumn{2}{|c|}{ Cordyla pinnata } & \multicolumn{2}{|c|}{ Detarium microcarpum } & \multicolumn{2}{|c|}{ Detarium senegalense } \\
\hline & $\mathrm{Tp}$ & $\mathrm{He}$ & $\mathrm{Tp}$ & $\mathrm{He}$ & $\mathrm{Tp}$ & $\mathrm{He}$ \\
\hline Parasitoses intestinales & 0,04 & 0,39 & 0,01 & 0,02 & & \\
\hline Maux de ventre & & 0,04 & & & & \\
\hline Difficultés à digérer & & 0,03 & & & & \\
\hline Dermatoses (kouli) & & 0,03 & 0,12 & 0,64 & 0,02 & 0,11 \\
\hline Hémorroïdes & 0,02 & & 0,03 & 0,09 & & \\
\hline Perte d'appétit & & 0,17 & & & & \\
\hline Problèmes de nerfs & & & & 0,16 & & \\
\hline Douleurs articulaires et Rhumatismes & 0,01 & & 0,01 & 0,11 & & \\
\hline Fièvre & & 0,03 & & & & \\
\hline Brulures & & & 0,02 & & & \\
\hline Plaies & & & 0,02 & & 0,02 & \\
\hline Difficultés à uriner (homme et la femme) & 0,01 & & 0,02 & & & \\
\hline Enurésie (enfant qui fait pipi au lit) & & & & & 0,02 & \\
\hline Enflures & 0,02 & 0,04 & & & 0,02 & \\
\hline Conjonctivite & 0,01 & & & & & \\
\hline Cancer & & & & & 0,02 & \\
\hline Défaillance système immunitaire & 0,01 & & & & & \\
\hline Prévention des épidémies (enfants) & & & 0,01 & & & \\
\hline Prévention contre les poisons & 0,01 & & & & & \\
\hline Carie dentaire et mauvaise haleine & & & 0,02 & & 0,02 & \\
\hline Hoquet & 0,01 & & & & & \\
\hline Problèmes menstruels & & & & & 0,02 & \\
\hline Problèmes de virilité chez l'homme & & & 0,01 & & & \\
\hline Asthme & 0,01 & & & & & \\
\hline Longues maladies & & & 0,01 & & & \\
\hline Paralysie & & & 0,01 & & & \\
\hline Bourdonnements d'oreilles + vertiges et sursauts & 0,01 & & & & & \\
\hline Vomissements & 0,01 & & & & & \\
\hline Kystes & 0,01 & & & & & \\
\hline Diabète & & 0,04 & & & & \\
\hline Total & 0,16 & 0,76 & 0,26 & 1,02 & 0,17 & 0,11 \\
\hline
\end{tabular}

Tp: tradipraticiens ; He: herboristes 
l'appartenance socio-professionnelle. A ce titre, les herboristes identifient souvent leurs cantines à des «pharmacies " où chaque patient peut trouver ce dont il a besoin pour se soigner. Par contre, les récolteurs vendent essentiellement leurs récoltes aux herboristes et à des tradipraticiens établis dans les centres urbains.

Les tradipraticiens utilisent les plantes pour confectionner des médicaments remis aux patients. En échange, les patients locaux les rémunèrent généralement en argent, avec un maximum de 7,62 Euro. Ils peuvent aussi payer en nature (chèvre, poules ou mil) ou symboliquement par une poignée de sable. Quant aux patients étrangers, le paiement se fait uniquement en argent et la somme à payer dépend du type de maladie et de sa durée. Pour les longues maladies, les prix peuvent atteindre 144,34 Euro.

Dans les marchés de Kaolack et de Dakar, la commercialisation se fait par bottes d'environ 144 grammes pour les écorces de Cordyla pinnata et 155 grammes pour les racines. Les bottes d'écorces de Detarium microcarpum pèsent environ 156 grammes tandis que les racines font environ 105 grammes. La collecte n'a pas permis de disposer d'échantillons d'écorces et de racines de Detarium senegalense chez les herboristes qui n'en disposaient pas au moment de l'enquête.

Les prix moyens d'achat pour $100 \mathrm{~g}$ montrent de légères différences en fonction des espèces, des parties et de la localité. Les herboristes achètent les produits chez les récolteurs à un prix compris entre 0,052 et 0,079 Euro/100g pour les écorces et entre 0,069 et 0,108 Euro/100g pour les racines (Tableau 3).

A leur tour, les herboristes commercialisent $100 \mathrm{~g}$ d'écorces à un prix compris entre 0,108 et 0,212 Euro. Les racines sont vendues entre 0,108 et 0,290 Euro/100g. Les prix de la tasse remplie de poudre obtenue à partir des écorces sont plus élevés; soit 1,046 Euro/100g de poudre de Cordyla pinnata et 1,524 Euro/100g de poudre de Detarium microcarpum. Le client choisit la nature du produit en fonction des prescriptions obtenues ou bien en fonction de sa simple préférence. Dans l'ensemble, les prix de vente sont plus élevés à Dakar qu'à Kaolack.

\subsection{Forces, faiblesses, opportunités et menaces de la fi- lière}

Les produits à usage médicinal des trois espèces font l'objet d'une forte demande à tous les maillons de la filière (Tableau 4).

La principale force recensée chez les acteurs de cette filière concerne leur étroite collaboration, notamment dans l'écoulement des produits. Cette collaboration se traduit également par la transmission de certaines connaissances sur les vertus médicinales des espèces indigènes et par la solidarité dans la vie sociale. En effet, les membres de l'association des herboristes se prêtent main forte dans les situations heureuses tout comme dans celles qui le sont moins. La possession de plusieurs récolteurs dans différentes régions est également une force dans la mesure où elle permet d'assurer la régularité des approvisionnements en produits médicinaux et de réduire les risques de rupture de stock.

Les opportunités de cette filière sont relatives à l'intérêt que lui portent les organisations de la société civile. En effet, le renforcement des capacités des acteurs par la société civile vise à mieux les professionnaliser par une meilleure organisation de la récolte et de la commercialisation des produits ainsi que par une meilleure prise en compte de l'hygiène et de la qualité des produits vendus. En dotant certains herboristes de kiosques, l'ONG Enda Santé leur permet d'entreposer leurs produits de sorte à réduire les risques de moisissures qui en entravent la qualité. En plus de cela, cette ONG a joué un rôle fédérateur en renforçant la collaboration entre acteurs.

Les principales faiblesses identifiées dans cette filière sont liées à l'exploitation anarchique de la ressource, à l'irrégularité des approvisionnements, aux retards dans les livraisons des produits, au non-respect de la règlementation forestière et à la faible représentation des femmes dans la filière. L'exploitation anarchique consiste en l'écorçage et à la recherche abusive de racines qui conduisent parfois à la mort de l'arbre. Elle est facilitée par le caractère discret du prélèvement des produits à usage médicinal. En effet, la récolte de produits à usage médicinal doit généralement obéir à certaines exigences et se faire à l'abri de regards indiscrets au risque de perdre l'efficacité du produit. Chez les récolteurs, le prélèvement de produits à usage médicinal tirés de Cordyla pinnata en saison des pluies est rendu difficile par la présence des paysans qui n'apprécient pas que des individus inconnus traversent leurs champs déjà cultivés pour écorcer des arbres ou pour rechercher des racines. La

Tableau 3: Fréquence d'approvisionnement, prix d'achat et de vente des produits médicinaux tirés des trois espèces/Table 3: Frequency of supply, purchase and sale prices of medicinal products from the three species.

\begin{tabular}{|c|c|c|c|c|c|c|c|c|c|c|c|}
\hline \multirow[t]{3}{*}{ Espèce } & \multirow{3}{*}{$\begin{array}{l}\text { Fréquence } \\
\text { d'appro- } \\
\text { vision- } \\
\text { nement }\end{array}$} & \multirow{3}{*}{$\begin{array}{l}\text { Nombre } \\
\text { de bottes } \\
\text { par achat } \\
\text { et par her- } \\
\text { boriste }\end{array}$} & \multicolumn{4}{|c|}{$\begin{array}{l}\text { Prix moyen d'achat en Euro et par } \\
100 \mathrm{~g}\end{array}$} & \multicolumn{4}{|c|}{$\begin{array}{l}\text { Prix moyen de vente en Euro et par } \\
100 g\end{array}$} & \multirow{3}{*}{$\begin{array}{l}\text { Prix de } \\
\text { vente en } \\
\text { EURO par } \\
100 g \\
\text { P o u d r e } \\
\text { d'écorces }\end{array}$} \\
\hline & & & \multicolumn{2}{|c|}{ Écorce } & \multicolumn{2}{|c|}{ Racine } & \multicolumn{2}{|c|}{ Écorce } & \multicolumn{2}{|c|}{ Racine } & \\
\hline & & & Kaolack & Dakar & Kaolack & Dakar & Kaolack & Dakar & Kaolack & Dakar & \\
\hline $\begin{array}{l}\text { Cordyla pin- } \\
\text { nata }\end{array}$ & $\begin{array}{l}\text { Hebdo- } \\
\text { madaire à } \\
\text { mensuel }\end{array}$ & 10 à 100 & 0,073 & 0,079 & 0,069 & 0,073 & 0,116 & 0,212 & 0,108 & 0,197 & 1,046 \\
\hline $\begin{array}{l}\text { Detarium } \\
\text { microcarpum }\end{array}$ & $\begin{array}{l}\text { Hebdoma- } \\
\text { daire }\end{array}$ & 15 à 200 & 0,052 & 0,073 & 0,098 & 0,108 & 0,108 & 0,195 & 0,162 & 0,290 & 1,524 \\
\hline
\end{tabular}


Tableau 4: Analyse des atouts et des contraintes de la filière des produits médicinaux tirés des trois espèces/Analysis of the strengths and constraints of the medicinal products value chain derived from the three species.

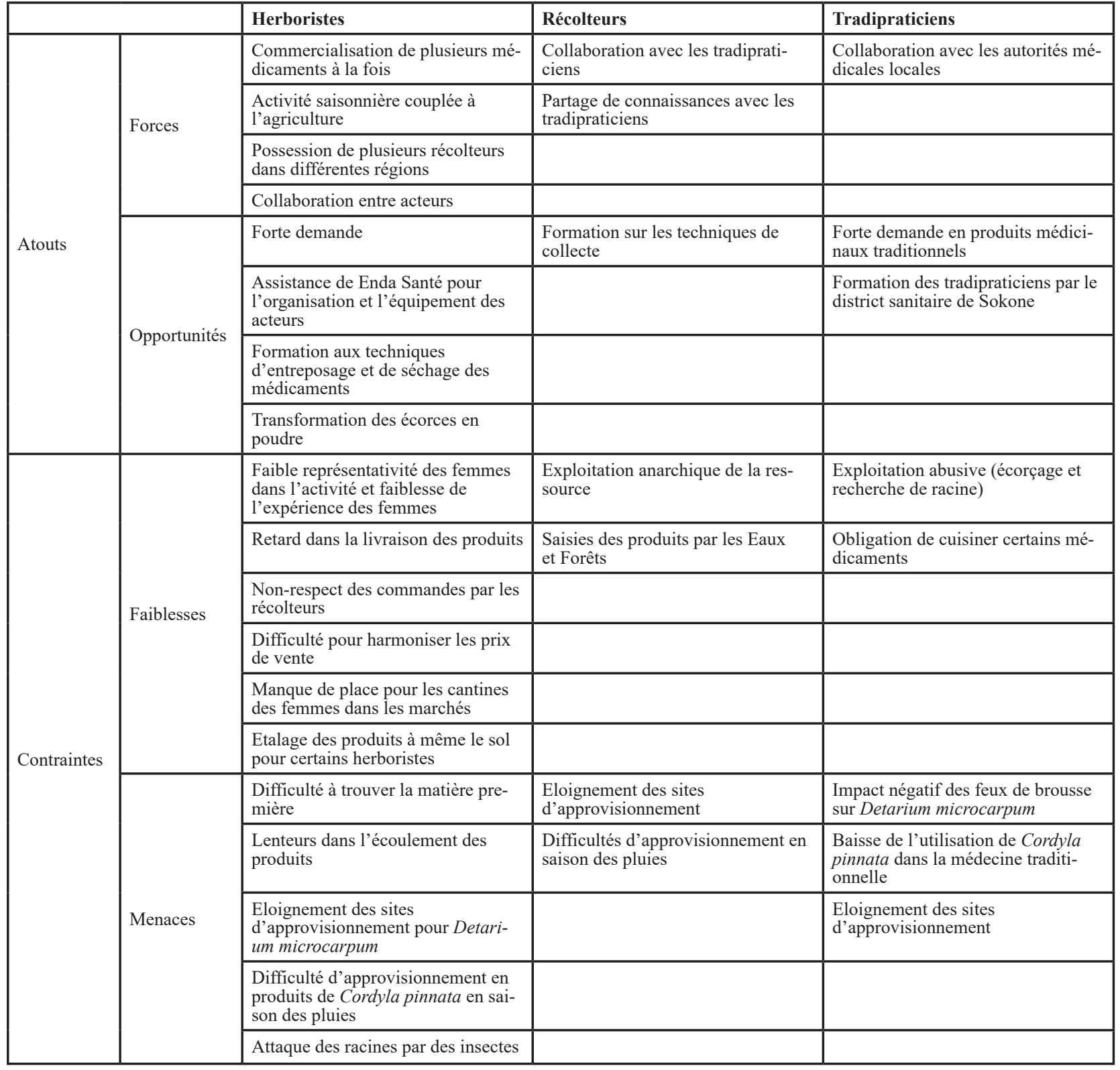

conséquence immédiate est qu'ils sont obligés de se rabattre sur les massifs forestiers protégés, dont la plupart sont éloignés des sites visités. Cela se répercute sur les délais de livraisons des produits aux herboristes situés en villes. Ces retards de livraisons s'expliquent également par la saisie de la marchandise par les services forestiers pour non-paiement des taxes et par la profession des récolteurs dont la plupart sont des agriculteurs et ne s'activent dans la récolte de produits à usage médicinale que durant la saison sèche. En plus de leur sous-représentation, il est ressorti de l'étude que les femmes ont une expérience faible en matière de commercialisation de produits à usage médicinal. Elles bénéficient par contre du soutien de leurs collègues hommes, membres de l'association des herboristes avec qui elles collaborent.

L'éloignement des sites d'approvisionnement surtout pour Detarium microcapum, l'inaccessibilité des arbres de Cordyla pinnata durant la saison des pluies, et la raréfaction de ces espèces liée surtout aux feux de brousse et aux activités anthropiques constituent des menaces qui entravent la vitalité de cette filière. Selon plusieurs herboristes interrogés à Kaolack et à Dakar, les produits issus de Cordyla pinnata et Detarium microcarpum proviennent de la zone frontalière avec la Gambie et au-delà

\section{Discussion}

Cette étude a montré que les acteurs impliqués dans la filière des produits tirés de Cordyla pinnata, Detarium microcarpum et Detarium senegalense sont surtout des hommes adultes (35-70 ans) et que les femmes sont peu représentées. Des tranches d'âge proches (30-70 ans) caractérisent également les tradipraticiens de la Mbanza-Ngungu en République Démocratique du Congo (BAKWAYe \& al. 2013). La faible représentation des femmes pourrait s'expliquer par le mode de transmission des connaissances locales qui se fait souvent de père en fils (ATAKPAMA $\&$ al. 2012) et par cer- 
taines exigences mystiques pour la collecte de certains produits à usage médicinal.

L'organisation de la filière des produits étudiés tourne autour de deux périodes et les prélèvements se font principalement dans les écosystèmes naturels et les agrosystèmes. L'approvisionnement en produits à usage médicinal dans les écosystèmes naturels est répandu au Sénégal (FAYE \&al. 1996) et au Bénin (Adomou \& al. 2012) où peu d'espèces médicinales sont domestiquées. Il se fait à une période où plusieurs espèces ligneuses sauvages bouclent le cycle de production fruitière avec la maturation de leurs fruits (GUEYE \& al. 2014). Les acteurs des différents maillons entretiennent des relations de coopération; qui leur permettent d'une part, d'acquérir de nouvelles connaissances et, d'autre part d'assurer la régularité de l'approvisionnement tel que décrit par AlbuQueRQue \& al. (2007).

L'importance des parties des espèces ligneuses diffère en fonction des réalités socio-culturelles et de l'espèce. Les résultats obtenus dans la présente étude montrent par exemple que pour Detarium microcarpum, les écorces et les racines sont les plus utilisées alors qu'au Mali, les feuilles de la même espèce sont plus employées dans la médecine traditionnelle (KouYatÉ \& al. 2009). Dans les pharmacopées Malawite (BUNDSCHUH \& al. 20111) et Namibienne (CHEIKHYOUSSEF \& al. 2011) et dans les étals du Bénin (ADOMOU $\&$ al. 2012), les racines et les feuilles sont plus utilisées de façon générale.

L'action antiparasitaire est plus connue pour Cordyla pinnata alors que Detarium microcarpum est plutôt connue pour le traitement des problèmes dermatologiques appelés localement kouli. Diverses espèces indigènes sont aussi utilisées, à l'image de Cordyla pinnata, dans le traitement des parasitoses intestinales. Au Burkina Faso, les racines de Sarcocephalus latifolius sont utilisées à cet effet mais avec un niveau de consensus inférieur $(\mathrm{CTU}=0,029)$ à celui trouvé dans la présente étude pour Cordyla pinnata (KABORÉ \& al. 2014). Par contre, chez Cordyla pinnata ce sont aussi bien les racines que les écorces qui sont utilisées pour traiter ces parasitoses. Les feuilles de Adansonia digitata sont aussi utilisées contre les parasites au Burkina Faso (SchumanN \& al. 2012).

Le terme générique kouli renferme un ensemble d'affections multiples dont la plupart se manifestent par des problèmes dermatologiques. Ces problèmes dermatologiques touchent le plus souvent la peau, les muqueuses et les phanères (ATIH 2018). Les résultats de LoubAKI \& al. (1999) ont en effet démontré que Detarium microcarpum a une grande importance médicinale avec une activité bactéricide contre plusieurs souches dont les staphylocoques. Cette importance peut être liée à l'acide kolavénique contenue dans ses feuilles et ses écorces et dont la forte inhibition de bactéries Gram-positif, telles que Bacillus subtilis et Staphylococcus aureus a été évoquée par CAVIN (2007). L'utilisation des parties de Detarium microcarpum contre les dermatoses avait déjà été signalée au Mali, chez les minianka (KouYATÉ $\&$ al. 2009). Au Sénégal, au Bénin et au Maroc, des espèces comme Carapa procera (GuEYE \& al. 2010), Opuntia ficusindica (El Hilah \& al. 2016) et Bombax costatum (AssogBA \& al. 2017) sont bien connues pour le traitement de dif- férents types de dermatoses. Les vertus de Detarium senegalense semblent mal connues et l'espèce est peu représentée dans les étals des herboristes interrogés. Les écorces de Detarium senegalense sont, entre autres, connues au Nigéria pour leur action antispasmodique, antibactérienne, anti-oxydante, astringente et sont utilisées pour traiter notamment les hémorroïdes, les blessures, les brûlures, les inflammations, la diarrhée, les maux de ventre, les rhumatismes, les maux de tête, les douleurs menstruelles et le choléra (Sowemimo \& al. 2011, UCHEGBU \& OKWU 2012). Les extraits de xyloglucane contenus dans les amandes de cette espèce auraient des propriétés prometteuses pour le traitement de maladies de santé publique comme le diabète et le cancer (WANG \& al. 1997 citant ElLis 1994); ce qui pourrait justifier l'avis de ce tradipraticien qui accorde à Detarium senegalense des vertus contre le cancer. Cependant, vu que certaines plantes ont des composés toxiques (FALL et al. 2017), il convient de renforcer l'encadrement de cette filière.

Dans le domaine de la commercialisation, les résultats montrent, de façon générale, une harmonisation des prix pour les produits tirés des trois espèces et une différence dans les prix de vente pratiqués dans la capitale sénégalaise et les villes secondaires. Cette différence s'explique notamment par l'existence de réglementations propres aux acteurs de chaque marché et par les efforts de structures d'encadrement de la société civile pour harmoniser les prix de vente des produits médicinaux. Les montants plus élevés notés dans la capitale pourraient s'expliquer par les prix de transports liés à l'éloignement des sites de récolte ainsi que par la plus forte demande (DrAmÉ 2003).

En dépit de la forte demande dont ces produits font l'objet, de l'encadrement des acteurs de la filière et de la collaboration qu'ils entretiennent, il a été noté plusieurs menaces et faiblesses dans la filière des produits à usages médicinal tirés de Cordyla pinnata, Detarium microcarpum et Detarium senegalense. Parmi les menaces identifiées, la raréfaction de certaines espèces et l'éloignement des sites d'approvisionnement ont été évoquées. Cette raréfaction, également signalée par BuNDSCHUH \& al. (2011), est en relation avec la perte de biodiversité notée en Afrique (IPBES, 2018) et dont les impacts se manifestent dans plusieurs aspects de la vie des communautés. En effet, en plus du fait que la plupart des prélèvements en plantes médicinales se font dans les formations végétales naturelles, l'effet de la commercialisation qui s'en suit induit souvent une surexploitation (Cunningham \& Mbenkum 1993, FAO année indéterminée); ce qui constitue un risque pour la pérennité et la disponibilité de certaines espèces (OMS 2003). Dans la zone concernée par cette étude, cette exploitation anarchique est surtout observée sur les populations de Detarium microcarpum car les écorces et les racines sont très sollicitées dans la médecine traditionnelle. Cette forte demande avait déjà été soulignée par SAMB (2002). L'exploitation anarchique pourrait également contribuer à la raréfaction de l'espèce dans la plupart des formations végétales qui sont proches des grandes villes du pays et à l'obligation pour les récolteurs d'aller dans les forêts jouxtant les pays limitrophes pour s'approvisionner. Ainsi, les récolteurs d'écorces et de racines sont souvent indexés par certains tradiprapraticiens dans les villages pour expliquer l'exploitation abusive 
de Detarium microcarpum à l'intérieur de la Forêt Classée de Patako et la modification de la structure de sa population qui était pourtant stable moins de vingt ans plus tôt (SAMBOU 2004).

L'importance des parties exploitées peut entraver la durabilité de la ressource. Dans cette étude, ce sont les écorces et les racines qui sont les plus utilisées. Or, il a été prouvé que le type de parties et les quantités prélevés ainsi que les méthodes d'extraction ont un impact sur la dynamique et la durabilité des peuplements naturels (Peters 1997, GuedJE Chaungueu 2000). Ainsi, l'exploitation des feuilles demeure moins nocive pour les plantes que celle des racines et des écorces, lorsqu'elle est bien pratiquée (KouYATÉ \& al. 2009). Il est aussi admis que la capacité de cicatrisation des arbres après écorçage dépend de l'espèce, de la superficie écorcée et du diamètre du tronc de l'arbre (GUEDJE CHAUNGUEU 2000). Chez Cedrelopsis grevei, à Madagascar, une cicatrisation complète a été notée dans $84 \%$ des cas, alors qu'elle a été partielle lorsque le cambium a été touché (ANDRIANOELISOA 2013). Un écorçage sur toute la circonférence de l'arbre entraine une destruction du système vasculaire et provoque sa mort (Guedje Chaungueu 2000). Cependant, lorsque le tronc est totalement coupé à la hauteur du pied, à peu près comme cela a été observé dans la Forêt Classée de Patako avec Detarium microcarpum (DIENG \& al. 2016), le développement de rejets peut être noté tel que décrit par Guedje Chaungueu (2000). Or, puisque cette espèce régénère bien par rejets de souches (KOUYATÉ 2005), l'élaboration et la mise en œuvre d'un plan de gestion participatif de la Forêt Classée de Patako devrait envisager une rotation dans son exploitation afin de lui permettre de se régénérer. L'application et la vulgarisation de techniques de récolte moins nocives pour l'arbre peuvent aussi être envisagées comme cela est recommandé pour Cedrelopsis grevei pour laquelle un écorçage en bandes proportionnelles au diamètre du tronc de l'arbre est préconisé (ANDRIANOELISOA 2013).

\section{CONCLUSION}

Le fonctionnement de la filière étudiée est lié au calendrier agricole du Sénégal. La récolte des produits à usage médicinal est généralement une activité d'appoint, qui permet aux acteurs impliqués d'avoir des sources de revenus complémentaires à l'agriculture; ce qui a des répercussions sur toute la filière. La récolte et la commercialisation des différentes parties de Cordyla pinnata, Detarium microcarpum et Detarium senegalense est essentiellement assurée par les hommes. Cependant, les interventions d'une ONG ont permis de renforcer les capacités des femmes en vue de leur meilleure implication dans cette filière. Les utilisations médicinales de Cordyla pinnata, Detarium microcarpum et Detarium senegalense sont diversifiées et concernent principalement les écorces, les racines et les feuilles qui sont, dans l'ensemble prélevés dans les écosystèmes naturels et dans les agrosystèmes. A la lumière de la dégradation des écosystèmes naturels, surtout forestiers notée ces dernières années au Sénégal et ailleurs en Afrique, cette étude permet de s'interroger sur la durabilité de cette filière et sur les impacts potentiels de la dégradation des écosystèmes naturels sur la santé des populations. En effet, l'exploitation à gran- de échelle des parties vitales des plantes ainsi que les mauvaises pratiques compromettent la survie des arbres et constituent une menace pour le bon fonctionnement de la filière. Afin de mieux tirer profit de ces ressources médicinales, il convient d'appuyer les actions de certaines ONG ainsi que la recherche scientifique pour une meilleure domestication de certaines espèces de plantes utilisées dans la médecine traditionnelle. Des recherches devraient également être menées afin d'identifier des seuils d'exploitation durables pour les principales parties des espèces végétales utilisées dans la médecine traditionnelle.

\section{Remerciements}

Ce travail a été réalisé dans le cadre du projet UNDESERT «Understanding and Combating Desertification to Mitigate its Impact on Ecosystem Services » (EU FP7 243906).

\section{REFERENCES}

Adomou AC, Yedomonhan H, Djossa B, Legba SI, OuMOROU M \& AKOEGNINOU A (2012): Etude Ethnobotanique des plantes médicinales vendues dans le marché d'AbomeyCalavi au Bénin. Int J Biol Chem Sci 6: 745-772.

Albugueroue UP, Monteiro JM, Ramos MA \& DE AmoRIM ELC (2007): Medicinal and magic plants from a public market in northeastern Brazil. J Ethnopharmacol 110: 7691.

ANDRIANOELISOA H (2013): La valorisation et l'exploitation non destructive des écorces de Cedrelopsis grevei. Rôle et place des transferts de gestion des ressources naturelles renouvelables dans les politiques forestières actuelles à Madagascar. CIRAD-00933941: 8 p.

ANSD (2015): Situation économique et sociale régionale 2013. SRSD de Dakar, 129 p.

ArbonNiER M (2019): Arbres, arbustes et lianes des zones sèches d'Afrique de l'Ouest. Quæ, Versailles, 776 p.

Assogba GA, Fandohan AB, Salako VK, Assogbadjo AE (2017): Usages de Bombax costatum (Malvaceae) dans les terroirs riverains de la réserve de biosphère de la Pendjari, République du Bénin. Bois For Trop 333 (3): 17-29.

Assogbadjo AE, Kakaï RG, Adjallala FH, Azihou F, Vodouhê GF, Kyndt T, Thimothée J \& Codja C (2010): Ethnic differences in use value and use patterns of the threatened multipurpose scrambling shrub (Caesalpinia bonduc L.) in Benin. J Med Plant Res 5: 1549-1557.

atakpama W, Batawila K, Dourma M, Pereki H, Wala K, Dimobe K, Akpagana K \& Gbeassor M (2012): Ethnobotanical knowledge of Sterculia setigera Del. in the sudanian zone of Togo (West Africa). ISRN Botany 2012: 8p.

ATIH (2018): Classification statistique internationale des maladies et des problèmes de santé connexes, Volume 1, Table analytique Édition 2018, 927p.

Bakwaye FN, Termote C, Kibungu K \& Van Damme O (2013): Identification et importance locale des plantes médicinales utilisées dans la région de Mbanza-Ngungu, République Démocratique du Congo. Bois For Trop 316(2): 6377.

BoOKer A, Johnston D \& HeInRICH M (2012): Value chains of herbal medicines-Research needs and key challenges in the context of ethnopharmacology. J Ethnopharmacol 140(2012): 624-633. 
Bundschun TV, Hahn K \& Wittig R (2011): The Medicinal Plants of the Woodlands in northern Malawi (Karonga District). Flora Veg Sudano-Sambesica 14: 3-8.

CAVIN AL (2007): Contribution à la connaissance taxonomique et chimique de fruits africains du genre "Detarium" (Fabaceae - Caesalpinioideae): "D. microcarpum" Guill. et Perr. et des formes comestibles et toxiques de "D. senegalense" J.F. Gmel. Thèse de doctorat: Université de Genève, 2007, no. Sc. 3838.

Cheikhyoussef A, Shapi M, Matengu K \& Ashekele HM (2011): Ethnobotanical study of indigenous knowledge on medicinal plant use by traditional healers in Oshikoto region, Namibia. J Ethnobiol Ethnomed 7: 10 p.

Christensen SN (2010): Socio-economic and ecological determinants of local scale tree distribution, diversity and dynamics in agro ecosystems in West-central Senegal. Master thesis, Aarhus University, 19 p.

Coppen JJW (1995): Gums, resins and latexes of plant origin and latexes. Non-wood forest products 6. FAO, Rome, $152 \mathrm{p}$.

Cunningham AB \& Mbenkum FT (1993): Sustainability of harvesting Prunus africana bark in Cameroon. A medicinal plant in international trade. UNESCO, Paris, $28 \mathrm{p}$.

De Wet H, Van Wyk BE (2008): An ethnobotanical survey of southern African Menispermaceae. S Afr J Bot 74: 2-9.

Dibong SD, Mvogo Ottou PB, Vandi D, Ndjib RC, MonKAM TChamaha F, Mrondo MPONDo E (2015): Ethnobotanique des plantes médicinales anti-hémorroïdaires des marchés et villages du Centre et du Littoral Cameroun. J Appl Biosci 96: 9072-9093.

DieNG SD (2017): Evaluation des services écosystémiques de Cordyla pinnata (Lepr. Ex A.Rich.) Milne-Redh., Detarium microcarpum Guill. et Perr. et Detarium senegalense (J.F. Gmel.) de la Forêt Classée de Patako et de ses environs (Centre-Ouest du Sénégal). Thèse de doctorat en Sciences de l'Environnement, Faculté des Sciences et Techniques, Université Cheikh Anta Diop de Dakar, 184 p+ Annexes.

Dieng SD, Diop M, Goudiaby A, Niang-Diop F, Faye LC, Guiro I, Sambou S, Lykke AM, Sambou B (2016): Caractérisation des services écosystémiques fournis par Cordyla pinnata dans la périphérie de la Forêt classée de Patako au Sénégal. VertigO, 16(2).

Dieng SD, Diop M, Faye LC, Goudiaby A, Niang-Diop F, Guiro I, Sambou S, Lykke AM, Sambou B (2016): Characterization of Ecosystem Services Provided by Detarium microcarpum around the Protected Forest of Patako (Senegal). J Appl Environ Biol Sci 6(12): 41-49.

Diop M, Sambou B \& Ly B (2012): Représentations de la forêt et répercussions sur la gestion des ressources forestières au Sénégal. VertigO, 12(2).

Diop N (2013): Caractérisation du ditax (Detarium senegalense J.F.Gmel) et étude de sa transformation en nectar. Thèse de doctorat, spécialité Systèmes Energétiques et Environnement, Université Cheikh Anta Diop de Dakar, 148p.

DiatTa CD, GueYe M \& AKro LE (20123): Les plantes médicinales utilisées contre les dermatoses dans la pharmacopée Baïnounk de Djibonker, région de Ziguinchor (Sénégal). J Appl Biosci 70: 5599-5607

DRAMÉ YY (2003): Contribution à la valorisation et à la conservation « ex situ » de deux plantes de la pharmacopée sénégalaise: Cordyla pinnata (Lepr.) Miln.Red. (Caesalpiniaceae)-Sterculia setigera Del. (Sterculiaceae).
Enquêtes socio-économiques, essais botaniques et pharmacognosiques de qualité. Thèse de Doctorat d'Etat, Faculté de médecine, de pharmacie et d'odontologie, $76 \mathrm{p}+$ annexes.

ELLIS PR (1994): Polysaccharide gums: their modulation of carbohydrate and lipid metabolism and role in the treatment of diabetes mellitus. In Gums and Stabilisers for the Food Industry 7, ed. G.O. Phillips, P.A. Williams and D.J. Wedlock, pp. 207-216. Oxford University Press, Oxford.

El hilah F, Ben Akka F, Bengueddour R, Rochdi A \& ZIDANE L (2016): Étude ethnobotanique des plantes médicinales utilisées dans le traitement des affections dermatologiques dans le plateau central marocain. J Appl Biosci 98: 9252-9260.

Fall AD, Bagla VP, Bassene E \& Eloff JN (2017): Phytochemical screening, antimicrobial and cytotoxicity studies of ethanol leaf extract of Aphania senegalensis (Sapindaceae). Afr J Tradit Complement Altern Med 14(4): 135-139.

FAO (année indéterminée): Trade in medicinal plants. Raw materials, Tropical and Horticultural service Commodities and Trade Division, Economic and Social Department, Rome, 62 p. ftp://ftp.fao.org/docrep/fao/008/af285e/ af $285 \mathrm{e} 00$.pdf. Consulté en ligne le 11 octobre 2015.

Faye B, Bassène E, Diouf M (1996): Phase expérimentale de production de phytomédicaments. In RejDali M, Birou K (1996): Diversité biologique et valorisation des plantes médicinales. Eds CTES. Proceedings. Rencontre francophone de coopération et de partenariat, 4-8 septembre 1995, Rabat, Maroc, 135-138.

GTZ (2007): Manuel de ValueLinks. La méthodologie de la promotion de la chaîne de valeur ajoutée. Première édition, $255 \mathrm{p}$.

Guedje Chaungueu NM (2000): Exploitation et gestion des produits forestiers non-ligneux (PFNL): l'exemple de Garcinia lucida Vesque. Communication pour le Workshop de CARPE du 18 au 20 juillet 2000, Limbé, 10 p.

Gueye M, Ayessou NC, Koma S, Diop S, Akpo LE \& Samb PI (2014): Wild fruits traditionally gathered by the Malinke ethnic group in the edge of Niokolo Koba Park (Senegal). Am J Plant Sci 5: 1306-1317.

Gueye M, Cissé A, Diatta CD, Diop S \& Koma S (2012): Etude ethnobotanique des plantes utilisées contre la constipation chez les Malinké de la communauté rurale de Tomboronkoto, Kédougou (Sénégal). Int J Biol Chem Sci 6: 773-781.

Gueye M, Kenfack D \& Forget PM (2010): Importance socio-culturelle, potentialités économiques et thérapeutiques du Carapa (Meliaceae) au Sénégal. In: van der BURGT X., van der Maesen J \& Onana JM (eds), Systématique et Conservation des Plantes Africaines, 359-367. Royal Botanic Gardens, Kew.

Heinrich M, Ankli A, Frei B, Weimann C \& Sticher O (1998): Medicinal plants in Mexico: healers' consensus and cultural importance. Soc Sci \& Med 47: 1859-1871.

IPBES (2019): Communiqué de presse: le dangereux déclin de la nature: un taux d'extinction des espèces «sans précédent » et qui s'accélère. Paris, France.

Kaboré SA, Hien M, OuÉdraogo D, Diallo TRE, Hahn K \& NACro HB (2014): Use of ecosystem services of Sarcocephalus latifolius (Sm.) E.A.Bruce and induced effect of human pressure on the species in the southwestern region of Burkina Faso. Ethnobot Res Appl 12: 561-570. 
Kerharo J \& AdAm JG (1974): La pharmacopée traditionnelle: plantes médicinales et toxiques. Vigot Frères, Paris, $1011 \mathrm{p}$.

Kouyaté AM, Meyer A, Van Damme P \& Diawara H (2009): Usages magico-médicinaux et vétérinaires de Detarium microcarpum (Fabaceae) au sud du Mali. In: van der Burgt X, van der Maesen J \& Onana JM (eds), Systematics and Conservation of African Plants. Royal Botanic Gardens, Kew, 367-374.

KOUYATÉ IR. AM (2005): Aspects ethnobotaniques et étude de la variabilité morphologique, biochimique et phénologique de Detarium microcarpum Guill. \& Perr. au Mali. Thèse de $\mathrm{PhD}$ en Biosciences Ingénieurs, Section Agronomie, Université de Gent, Belgique, $190 \mathrm{p}$

LOUbaki BC, Ouattara AS, Ouattara CAT, OuÉDraogoTraOré R \& TraORÉ AS (1999): Activités antimicrobiennes des extraits aqueux totaux de Detarium microcarpum $[\mathrm{Ce}-$ salpinaceae (Guill et Perr)] sur huit espèces bactériennes impliquées dans certaines maladies infectieuses au Burkina Faso. Rev CAMES 01: 66-73.

LYKKe AM, KRISTENSEN MK \& GANABA S (2004): Valuation of local use and dynamics of 56 woody species in the Sahel. Biodivers Conserv 13: 1961-1990.

Martín-López B, García-Llorente M, Palomo I \& MonTES C (2011): The conservation against development paradigm in protected areas : Valuation of ecosystem services in the Doñana social-ecological system (southwestern Spain). Ecol Econ 70: 1481-1491.

Monteiro JM, Albuquerque UP, Lins-Neto EMF, AraúJo EL\& AMORIM ELC (2006): Use patterns and knowledge of medicinal species among two rural communities in Brazil's semi-arid northeastern region. J Ethnopharmacol 105(1-2): 173-186.

OMS (2003): Médecine traditionnelle. Aide-mémoire n 134 , http://www.who.int/mediacentre/factsheets/2003/ fs $134 /$ fr/. Consulté le 7 octobre 2015.

Peters CM (1997): Exploitation soutenue de produits forestiers autres que le bois en forêt tropicale humide: manuel d'initiation écologique. Série générale d'appui à la biodiversité 2. WWF-NC-WRI/ USAID, Washington, 49 p.

Roussel J (1995): Pépinières et plantations forestières en Afrique tropicale sèche: manuel à l'usage des ingénieurs et techniciens du reboisement. Dakar, Sénégal, ISRA/CIRAD, $435 \mathrm{p}$.
RusSEL BH (2006): Research methods in anthropology. Qualitative quantitative approches. Fourth edition, Alta Mira Press, Oxford, 824 p.

SAmB AH (2002): Contribution à la conservation « ex situ » et à la valorisation de deux espèces de la pharmacopée sénégalaise: Detarium microcarpum Gill et Perr. (Ceasalpiniaceae); Tinospora bakis (A. Rich.) Miers (Menispermaceae). Thèse de Doctorat d'Etat, Faculté de médecine, de pharmacie et d'odontologie, Université Cheikh Anta Diop, Dakar, 104 p. + annexes.

SAmbou B (2004): Evaluation de l'état, de la dynamique et des tendances évolutives de la flore et de la végétation ligneuses dans les domaines soudanien et sub-guinéen au Sénégal. Thèse de Doctorat d'Etat. Faculté des Sciences et Techniques, Université Cheikh Anta Diop, Dakar, 210 p.

Schumann K, Wittig R, Thiombiano A, Becker U \& HAHN K (2012): Uses, management, and population status of the baobab in eastern Burkina Faso. Agrofor Syst 85: 263-278.

Sieglstetter R, Hahn K \& Wittig R (2011): The use of woody species in northern Benin. Flora Veg Sudano-Sambesica 14: 19-23.

Sowemimo AA, Рendota C, Окон B, Омотоsho T, Idika N \& AdeKunle AA (2011): Chemical composition, antimicrobial activity, proximate analysis and mineral content of the seed of Detarium senegalense JF Gmelin. Afr J Biotech 10: 9875-9879.

UCHEGBU RI \& OKwU DE (2012): An evaluation of the phytochemical and nutrient composition of the seeds and stem bark of Detarium senegalense Gmelin. J Nat Sci Res 2: 107112.

Vodouhê FG, Coulibaly O, Assogbadjo AE \& Sinsin B (2008): Medicinal plant commercialization in Benin: an analysis of profit distribution equity across supply chain actors and its effect on the sustainable use of harvested species. J Med Plant Res 2(11): 331-340.

Wang Q, Ellis PR, Ross-Murphy SB, Burchard W (1997): Solution characteristics of the xyloglucan extracted from Detarium senegalense Gmelin. Carbohydr Polym 33: 115-124.

Wittig R, Dingermann T, Sieglstetter R, Xie Y, ThiomBIANO A,\& HAHN K (2013): World-wide every fifth vascular plant species is or was used as medicinal or aromatic plant. Flora Veg Sudano-Sambesica 16: 3-9. 